\title{
Characterizations of Rad-supplemented modules
}

\author{
Ergül Türkmen and Ali Pancar
}




\title{
CHARACTERIZATIONS OFRad-SUPPLEMENTED MODULES
}

\author{
ERGÜL TÜRKMEN AND ALI PANCAR
}

Received 15 September, 2011

\begin{abstract}
We prove that a commutative ring $R$ is an artinian principal ideal ring if and only if the ring is semilocal and every Rad-supplemented $R$-module is a direct sum of w-local $R$-modules. Moreover, we study of extensions of Rad-supplemented modules over commutative noetherian rings, and we show that if $\frac{M}{N}$ is reduced, $M$ is Rad-supplemented if and only if $N$ and $\frac{M}{N}$ are Rad-supplemented. We also prove that over a dedekind domain an indecomposable, amply Rad-supplemented radical module is hollow radical.
\end{abstract}

2000 Mathematics Subject Classification: 16G10; 16D10; 16D99

Keywords: Rad-supplement, Rad-supplemented module, extension, semilocal ring, artinian principal ideal ring

\section{INTRODUCTION}

In this note $R$ will be an associative ring with identity. Unless otherwise mentioned, all modules will be unital left $R$-modules. Let $R$ be such a ring and $M$ be an $R$-module. The notation $N \subseteq M$ means that $N$ is a submodule of $M$. A submodule $S$ of $M$ is called small in $M$, denoted by $S<<M$, if $S+N \neq M$ for every proper submodule $N$ of $M$. We denote by $\operatorname{Rad}(M)$ the radical of $M$. A non-zero module $M$ is called hollow if every proper submodule of $M$ is small in $M$, and it is called local if it is hollow and $\operatorname{Rad}(M)$ is a maximal submodule of $M$. Let $M$ be a module. $M$ is called supplemented if every submodule $N$ of $M$ has a supplement, that is a submodule $K$ of $M$ minimal with respect to $N+K=M$. Equivalently, $N+K=M$ and $N \cap K<<K$ ([12]). Following [12], $M$ is called amply supplemented if, for any two submodules $U$ and $V$ of $M$ with $U+V=M, V$ contains a supplement of $U$ in $M$. Clearly, hollow modules are amply supplemented and amply supplemented modules are supplemented.

Recall from Lomp [7] that a module $M$ is said to be semilocal if $\frac{M}{\operatorname{Rad}(M)}$ is semisimple, and a ring $R$ is said to be semilocal if it is semilocal as a left (right) module over itself. It is shown in [7, Teorem 3.5] that a ring $R$ is semilocal if and only if every left $R$-module is semilocal.

As a proper generalization of supplemented modules, the notion of Rad-supplemented modules, which has been introduced by Xue [13], has been studied recently 
(see [1,4,5]). Let $M$ be a module and $N$ be a submodule of $M$. A submodule $K$ of $M$ is called a Rad-supplement of $N$ in $M$ (according to [13], generalized supplement) if $N+K=M$ and $N \cap K \subseteq \operatorname{Rad}(K)$. Since $\operatorname{Rad}(K)$ is the sum of all small submodules of $K$, every supplement submodule is a $\operatorname{Rad}$-supplement in $M$. A module $M$ is called Rad-supplemented (according to [13], generalized supplemented) if every submodule $N$ of $M$ has a Rad-supplement $K$ in $M$, and it is called amply $\mathrm{Rad}$-supplemented (according to [13], generalized amply supplemented) if every submodule $N$ of $M$ has ample Rad-supplements in $M$, i. e., $N+L=M$ implies that $N$ has a $\operatorname{Rad}$-supplement $K \subseteq L$. In [5], the various properties of $\operatorname{Rad}$ supplemented modules are extensively studied. In addition, it is shown in [1, 2.2.(2) and 2.3.(3)] that factor modules of a Rad-supplemented module and finite sums of Rad-supplemented modules are Rad-supplemented. It is of obvious interest to investigate extensions and characterizations of Rad-supplemented modules. This is the focus of our investigations in this paper.

Let $\Gamma$ be a class of modules and let $0 \rightarrow N \rightarrow M \rightarrow K \rightarrow 0$ be any short exact sequence. Here $M$ is an extension of $N$ by $K$ and $\Gamma$ is called closed under extensions if $N, K \in \Gamma$ implies $M \in \Gamma$. It is clear that, for modules $N \subseteq M, M$ is an extension of $N$.

In this article, we prove that a commutative ring $R$ is an artinian principal ideal ring if and only if the ring is semilocal and every Rad-supplemented $R$-module is a direct sum of w-local $R$-modules if and only if every left $R$-module is a direct sum of w-local $R$-modules. We give a characterization of semisimple rings via Radsupplements. We show that a semilocal ring $R$ is left perfect if and only if every Rad-supplemented module is (generalized) semiperfect. Some examples are given in order to show that the class of Rad-supplemented modules is not generally closed under extensions. Let $R$ be a commutative noetherian ring and $M$ be an $R$-module with $N \subseteq M$. If $\frac{M}{N}$ is reduced, $M$ is $\operatorname{Rad}$-supplemented if and only if $N$ and $\frac{M}{N}$ are Rad-supplemented. It follows that a ring $R$ is semilocal if and only if every left $R$-module with Rad-supplemented radical is Rad-supplemented. Over a dedekind domain a radical module is amply Rad-supplemented and indecomposable if and only if the module is hollow radical. Every indecomposable, w-local and amply Rad-supplemented module over a dedekind domain is local.

\section{Rad-SUPPLEMENTED MODULES OVER ANY RINGS}

Let $R$ be any ring and $M$ be an $R$-module. A submodule $N$ of $M$ is called radical if $N$ has no maximal submodules, i.e. $N=\operatorname{Rad}(N)$. Note that radical modules are Rad-supplemented. This fact plays a key role in our study. By $P(M)$ we denote the sum of all radical submodule of a module $M$. It is clear that, for any module $M$, $P(M)$ is the largest radical submodule and so $P(M)$ is Rad-supplemented. Using the mentioned facts, we give examples of a module, which is Rad-supplemented but not supplemented. We see, for example, the left $\mathbb{Z}$-module $M=\mathbb{Z} \mathbb{Q}$. 
Firstly we have the following lemma.

Lemma 1. Let $M$ be a module and $N \subseteq U \subseteq M$. Then $U$ is Rad-supplemented if and only if $\frac{U}{P(N)}$ is Rad-supplemented.

Proof. $(\Rightarrow)$ Let $U$ be Rad-supplemented. By [1, $2.2(2)], \frac{U}{P(N)}$ is Rad-supplemented as a factor module of $U$.

$(\Leftarrow)$ Let $U^{\prime}$ be any submodule of $U$. By the assumption, there exists a submodule $\frac{V}{P(N)}$ of $\frac{U}{P(N)}$ such that $\frac{U^{\prime}+P(N)}{P(N)}+\frac{V}{P(N)}=\frac{U}{P(N)}$ and

$$
\left(\frac{U^{\prime}+P(N)}{P(N)}\right) \bigcap\left(\frac{V}{P(N)}\right) \subseteq \operatorname{Rad}\left(\frac{V}{P(N)}\right) .
$$

Then $\left(U^{\prime}+P(N)\right)+V=U$ and hence $U^{\prime}+V=U$. Since $P(N)=\operatorname{Rad}(P(N)) \subseteq$ $\operatorname{Rad}(V)$, it follows that $\frac{U^{\prime} \cap V+P(N)}{P(N)}=\frac{\left(U^{\prime}+P(N)\right) \cap V}{P(N)}=\left(\frac{U^{\prime}+P(N)}{P(N)}\right) \cap\left(\frac{V}{P(N)}\right) \subseteq$ $\operatorname{Rad}\left(\frac{V}{P(N)}\right)=\frac{\operatorname{Rad}(V)}{P(N)}$, which means that $U^{\prime} \cap V \subseteq \operatorname{Rad}(V)$. So $V$ is a $\operatorname{Rad}$-supplement of $U^{\prime}$ in $U$. Hence $U$ is Rad-supplemented.

Corollary 1. Let $M$ be a module and $N$ be a submodule of $M . M$ is Radsupplemented if and only if $\frac{M}{P(N)}$ is $\mathrm{Rad}$-supplemented. In particular, $M$ is $\mathrm{Rad}$ supplemented if and only if $\frac{M}{P(M)}$ is $\mathrm{Rad}$-supplemented.

Proof. It follows from Lemma 1.

Recall from [5, Corollary 4.2] that if a submodule $V$ of a module $M$ is a Radsupplement in $M$, then $\operatorname{Rad}(V)=V \cap \operatorname{Rad}(M)$.

Now we shall show that the rings whose modules are Rad-supplement submodules in every extension are semisimple in the following theorem.

Theorem 1. Let $R$ be any ring. Then the following statements are equivalent.

(1) $R$ is semisimple.

(2) Every left $R$-module is a Rad-supplement in every extension.

(3) Every left $R$-module is a Rad-supplement in every injective extension .

(4) Every left ideal of $R$ is a $\mathrm{Rad}$-supplement in every injective extension.

Proof. (1) $\Rightarrow$ (2) Let $N$ be an $R$-module and $M$ be any extension of $N$. By the hypothesis and [6, Corollary 8.2.2 (a)], $M$ is semisimple, and so $N$ is a direct summand of $M$. It follows that $N$ is a $\operatorname{Rad}$-supplement in $M$.

(2) $\Rightarrow$ (3) $\Rightarrow$ (4) Clear.

(4) $\Rightarrow(1)$ Let $I$ be any left ideal of $R$. By the hypothesis, $I$ is a Rad-supplement in its injective hull $E(I)$. Then we have $I+J=E(I)$ and $I \cap J \subseteq \operatorname{Rad}(I)$ for some submodule $J \subseteq E(I)$. If $m \in I \cap J$, then $\operatorname{Rm} \subseteq \operatorname{Rad}(I) \subseteq \operatorname{Rad}(E(I))$. By (4), $R m$ is a $\operatorname{Rad}$-supplement in $E(I)$ and so $\operatorname{Rad}(R m)=R m \cap \operatorname{Rad}(E(I))=R m$. 
Therefore $m=0$. This means that $I \oplus J=E(I)$ and so $I$ is injective, and hence a direct summand of $R$. By [6, Corollary 8.2.2 (a)], $R$ is semisimple.

A ring $R$ is $\mathrm{Rad}$-supplemented if ${ }_{R} R$ (or $R_{R}$ ) is a $\mathrm{Rad}$-supplemented module. It is clear that semiperfect (i.e., supplemented) rings are Rad-supplemented. Characterizations of semiperfect rings have been studied extensively by many authors recently. Now we shall give a characterization of Rad-supplemented rings. Firstly, we need the following simple lemmas.

Lemma 2. Let $R$ be any ring with identity. Then $R$ is Rad-supplemented if and only if every cyclic R-module is Rad-supplemented.

Proof. Let $R$ be a Rad-supplemented ring. Suppose that $M$ is any cyclic $R$ module. Then there exists an element $m$ of $M$ such that $M=R m$. Note that $\frac{R}{\operatorname{Ann}(m)} \cong R m$, where Ann $(m)$ is the set of all elements $r$ of $R$ such that $r m=0$. From [1, 2.2.(2)] the hypothesis implies that $\frac{R}{\operatorname{Ann}(m)}$ is Rad-supplemented and so $R m$ is Rad-supplemented. The converse is clear.

Lemma 3. Let $M$ be a module with $U+V=M$ for submodules $U, V$ of $M$. If $V$ contains a Rad-supplement of $U$ in $M$, then $U \cap V$ has a $\operatorname{Rad-supplement~in~} V$.

Proof. Suppose that a submodule $K$ of $V$ is a $\operatorname{Rad}$-supplement of $U$ in $M$. Then, we have $U+K=M$ and $U \cap K \subseteq \operatorname{Rad}(K)$. From the modular law, $U \cap V+K=V$. Since $K \subseteq V$, then $(U \cap V) \cap K=U \cap K \subseteq \operatorname{Rad}(K)$. So $K$ is a $\operatorname{Rad}$-supplement of $U \cap V$ in $V$.

Theorem 2. The following statements are equivalent for any ring $R$.

(1) $R$ is Rad-supplemented.

(2) $R$ has ample Rad-supplements in every finitely generated extension.

(3) Every cyclic R-module has ample Rad-supplements in every finitely generated extension.

Proof. (1) $\Rightarrow$ (3) Let $N$ be any cyclic $R$-module and $M$ be any finitely generated extension of $N$. Since $R$ is Rad-supplemented, by Lemma 2, every cyclic submodule of $M$ is Rad-supplemented and so $M$ is amply Rad-supplemented by [11, Corollary 3.6]. Therefore $N$ has ample Rad-supplements in $M$.

(3) $\Rightarrow$ (2) It is obvious.

(2) $\Rightarrow$ (1) For any left ideal $I$ of $R$, consider the finitely generated pushout $R$ module $N=\frac{R \oplus R}{K}$, where $K$ is the set of all elements $k$ of $R \oplus R$ such that $k=$ $(r,-r)$ for all $r \in I$. Then there exist monomorphisms $f, g: R \rightarrow N$ such that $N=$ $f(R)+g(R)$. The hypothesis implies that $f(R)$ has a Rad-supplement $V$ in $N$ with $V \subseteq g(R)$. So, by Lemma 3, $V$ is a Rad-supplement of $f(R) \cap g(R)$ in $g(R)$. Note that $I=g^{-1}(f(R) \cap g(R))$. It follows that $R=I+g^{-1}(V)$ and $I \cap g^{-1}(V) \subseteq$ $\operatorname{Rad}\left(g^{-1}(V)\right)$. Hence $R$ is $\operatorname{Rad}$-supplemented. 
We say that a module $M w$-local if $\operatorname{Rad}(M)$ is a maximal submodule of $M$ as in [4]. Every local module is w-local. It is well known that a commutative ring $R$ has the property that every $R$-module is a direct sum of local $R$-modules if and only if $R$ is an artinian principal ideal ring. Now, we prove that if $R$ is a commutative ring and every $R$-module is a direct sum of w-local $R$-modules, then $R$ is an artinian principal ideal ring in the following theorem.

Theorem 3. The following are equivalent for a commutative ring $R$.

(1) Every left $R$-module is a direct sum of $w$-local $R$-modules.

(2) $R$ is semilocal and every Rad-supplemented left $R$-module is a direct sum of $w$-local $R$-modules.

(3) $R$ is an artinian principal ideal ring.

Proof. (1) $\Rightarrow$ (2) Write $\frac{R}{\operatorname{Rad}(R)}=\bigoplus_{i \in I} N_{i}$, where each $N_{i}$ is w-local. Since $\operatorname{Rad}\left(\frac{R}{\operatorname{Rad}(R)}\right)=0$, for all $i \in I, \operatorname{Rad}\left(N_{i}\right)=0$. So $N_{i}$ is simple. Thus $\frac{R}{\operatorname{Rad}(R)}$ is semisimple and so $R$ is semilocal. The rest of the proof is clear.

(2) $\Rightarrow$ (3) Let $F=R^{(\Lambda)}$ any index set $\Lambda$. Suppose that $\operatorname{Rad}\left(\frac{F}{N}\right)=\frac{F}{N}$ for some submodule $N$ of $F$. By the assumption, we can write $\frac{F}{N}=\bigoplus_{i \in I} M_{i}$ where $M_{i}$ is w-local for all $i \in I$. By [12, 21.6.(5)], $\operatorname{Rad}\left(\frac{F}{N}\right)=\bigoplus_{i \in I} \operatorname{Rad}\left(M_{i}\right)$ and so each $M_{i}$ is radical as a direct summand of $\frac{F}{N}$. Since $M_{i}$ is w-local, we obtain that, for all $i \in I, M_{i}=0$. Therefore $\frac{F}{N}=0$. This means that $\operatorname{Rad}(F)<<F$. It follows from $[12,43.9]$ that $R$ is left perfect. Applying $[12,43.9]$ again, we deduce that every left $R$-module is Rad-supplemented and so every left $R$-module is a direct sum of wlocal $R$-modules. If $N$ is aw-local, then $N$ is local because $R$ is left perfect. Hence every left $R$-module is a direct sum of cyclic $R$-modules. By [9, Theorem 6.7], $R$ is an artinian principal ideal ring.

(3) $\Rightarrow(1)$ is clear.

The following corollary is an immediate consequence of Theorem 3.

Corollary 2. Let $R$ be a commutative semilocal ring. Then, $R$ is an artinian principal ideal ring if and only if every $\mathrm{Rad}$-supplemented left $R$-module is a direct sum of $w$-local $R$-modules.

Let $f: P \rightarrow M$ be an epimorphism. Xue [13] calls $f$ a (generalized) cover if $(\operatorname{Ker}(f) \subseteq \operatorname{Rad}(P)) \operatorname{Ker}(f)<<P$, and calls a ( generalized) cover $f$ a (generalized) projective cover if $P$ is a projective module. In the spirit of [13], a module $M$ is said to be (generalized) semiperfect if every factor module of $M$ has a (generalized) projective cover. He [13, Theorem 2.2] proved that every generalized semiperfect module is Rad-supplemented. Now, we obtain the following result.

Proposition 1. Let $R$ be a semilocal ring. Every Rad-supplemented left $R$-module is (generalized) semiperfect if and only if $R$ is left perfect. 
Proof. $(\Rightarrow)$ Let $M=\operatorname{Rad}(M)$. Since $M$ is Rad-supplemented, it follows from the hypothesis that $M$ is generalized semiperfect. Then, there exists a generalized cover $f: F \rightarrow M$ with a projective module $F$. Since $\operatorname{Ker}(f) \subseteq \operatorname{Rad}(F) \neq F$, it follows that $M=0$. By [12, 43.9], $R$ is left perfect.

$(\Leftarrow)$ This is immediate.

\section{Rad-SUPPLEMENTED MODULES OVER COMMUTATIVE NOETHERIAN RINGS}

Throughout this section, unless otherwise stated, we shall consider commutative noetherian rings.

An $R$-module $M$ is called coatomic if every proper submodule of $M$ is contained in a maximal submodule of $M$, and it is called reduced if every submodule of $M$ contains a maximal submodule, that is, $P(M)=0$. Note that $\operatorname{Rad}(M)$ is small in $M$ for every coatomic $R$-module $M$.

Lemma 4. The following statements are equivalent for a $\mathrm{Rad}$-supplemented module $M$.

(1) $M$ is coatomic.

(2) $M$ is reduced.

(3) $\operatorname{Rad}(M)$ is small in $M$.

If the module $M$ satisfies one of the equivalent conditions, then $M$ is supplemented.

Proof. (1) $\Rightarrow$ (2) Let $M$ be a coatomic module. By [15, Lemma 1.1], every submodule of $M$ is coatomic and so $P(M)=0$, which means that $M$ is reduced.

(2) $\Rightarrow$ (3) Suppose that $M=\operatorname{Rad}(M)+N$ for some submodule $N$ of $M$. Then we can write $\operatorname{Rad}\left(\frac{M}{N}\right)=\frac{M}{N}$. Since $M$ is $\operatorname{Rad}$-supplemented, $N$ has a $\operatorname{Rad}$-supplement $V$ in $M$. From (2) it follows that $V$ has a maximal submodule $K$. So $\frac{K}{N \cap V}$ is a maximal submodule of $\frac{V}{N \cap V}$. Note that

$$
\frac{M}{N} \cong \frac{V}{N \cap V}
$$

contains a maximal submodule and thus $\frac{M}{N}=0$. Therefore $M=N$. This proves (3).

(3) $\Rightarrow$ (1) The assumption implies that, for any proper submodule $U \subseteq M$, there exists a submodule $V$ of $M$ such that $U+V=M$ and $U \cap V \subseteq \operatorname{Rad}(V)$. Since $\operatorname{Rad}(M)<<M, V$ is not contained in a maximal submodule $K$ of $M$. Then the submodule $U+V \cap K$ of $M$ is maximal. Thus $M$ is coatomic.

Suppose that Rad-supplemented module $M$ satisfies one of these conditions. Then $M$ is supplemented by [5, Proposition 7.3].

The following result follows from [5, Proposition 7.3]. We give this result as a consequence of Lemma 4.

Corollary 3. For a module $M, M$ is Rad-supplemented if and only if $\frac{M}{P(M)}$ is supplemented. 
A submodule of a Rad-supplemented module need not be Rad-supplemented, in general. To see this actuality, we shall consider the left $\mathbb{Z}$-module $M=\mathbb{Z} \mathbb{Q}$. It is well known that $M$ is Rad-supplemented. On the other hand, the submodule $\mathbb{Z} \mathbb{Z}$ of $M$ is not semisimple.

Now, we show that a submodule of a Rad-supplemented module is Rad-supplement ed under a certain condition.

Proposition 2. Let $M$ be a module and $N \subseteq M$. Suppose that $\frac{M}{N}$ is reduced. If $M$ is $\mathrm{Rad}$-supplemented, then $N$ is $\mathrm{Rad}$-supplemented.

Proof. According to [1, 2.2.(2)], $\frac{M}{N}$ is Rad-supplemented as a factor module of $M$. Since $\frac{M}{N}$ is reduced, $P\left(\frac{M}{N}\right)=0$. Therefore $\frac{M}{N}$ is supplemented by Lemma 4 . Since $M$ is Rad-supplemented, $\frac{M}{P(N)}$ is Rad-supplemented by Corollary 1 . Note that

$$
\frac{\frac{M}{P(N)}}{\frac{N}{P(N)}} \cong \frac{M}{N}
$$

is reduced and thus $\frac{M}{P(N)}$ is reduced by [14, Lemma 1.5 (a)]. It follows from Lemma 4 that $\frac{M}{P(N)}$ is supplemented. Thus $\frac{N}{P(N)}$ is supplemented by [8, Proposition 2.6]. So $\frac{N}{P(N)}$ is Rad-supplemented. Hence $N$ is Rad-supplemented by Lemma 1.

Using Proposition 2, we obtain the following result.

Corollary 4. The following statements are equivalent for any module $M$.

(1) $M$ is Rad-supplemented.

(2) Every maximal submodule of $M$ is Rad-supplemented.

(3) Every cofinite submodule of $M$ is Rad-supplemented.

Proof. (1) $\Rightarrow$ (3) If $N$ is a cofinite submodule of $M$, then $\frac{M}{N}$ is finitely generated and so $\frac{M}{N}$ is reduced. From Proposition 2, the proof follows.

(3) $\Rightarrow(2)$ is clear.

(2) $\Rightarrow$ (1) Let $M=M_{1}+M_{2}$, where $M_{1}$ and $M_{2}$ are maximal submodules of $M$. Since $M_{1}$ and $M_{2}$ are Rad-supplemented modules, $M$ is Rad-supplemented according to [1, 2.3.(3)]. If $M$ is w-local, $\operatorname{Rad}(M)$ is maximal and so $M=\operatorname{Rad}(M)+U$ for every proper submodule $U$ of $M$ with $U \nsubseteq \operatorname{Rad}(M)$. By [1, 2.3.(1)], $U$ has a $\operatorname{Rad}$-supplement in $M$ since $\operatorname{Rad}(M)$ is $\operatorname{Rad}$-supplemented. Hence $M$ is $\operatorname{Rad}$ supplemented.

The following example shows that the class of Rad-supplemented modules is not closed under extensions, in general.

Example 1. Let $\Lambda$ be a collection of maximal ideals of the noetherian commutative ring $\mathbb{Z}$. Suppose that $M$ is the left $\mathbb{Z}$-module $\prod_{\mathfrak{p} \in \Lambda}\left(\frac{\mathbb{Z}}{\mathfrak{p}}\right)$. Then $\operatorname{Rad}(M)=0$. By [3, Lemma 2.9], for some submodule $N$ of $M$, we have $\frac{N}{T} \cong \mathbb{Q}$, where $T$ is the 
direct sum of simple $\mathbb{Z}$-modules $\frac{\mathbb{Z}}{\mathfrak{p}}$. Then $N$ is an extension of $T$ by $\mathbb{Q}$. Since $T$ is semisimple, it is Rad-supplemented. On the other hand, the submodule $N$ is not Rad-supplemented.

Later we shall give another example of such modules (see Example 2).

Theorem 4. Let $0 \rightarrow N \rightarrow M \rightarrow K \rightarrow 0$ be a short exact sequence. Suppose that $K$ is reduced. Then $M$ is Rad-supplemented if and only if $N$ and $K$ are $\operatorname{Rad-}$ supplemented.

Proof. $(\Rightarrow)$ It follows from Proposition 2 and [1, 2.2.(2)].

$(\Leftarrow)$ By Lemma $4, K$ is supplemented. Since $N$ is Rad-supplemented, $\frac{N}{P(N)}$ is supplemented by Corollary 3. It follows from [8, Proposition 2.6] that $\frac{M}{P(N)}$ is $\operatorname{Rad}-$ supplemented. Hence $M$ is Rad-supplemented by Corollary 1 .

Corollary 5. A module $M$ is Rad-supplemented if and only if it is an extension of $a \mathrm{Rad}$-supplemented submodule by a reduced supplemented module.

Proof. If $M$ has no maximal submodules, the result is obvious as $\frac{M}{P(M)}=0$. Suppose that $M \neq P(M)$. Then this gives the existence of a reduced factor module of $M$. Therefore the assertion follows from Theorem 4 .

Proposition 3. Let $M$ be a module. $M$ is Rad-supplemented if and only if $M$ is semilocal and $\operatorname{Rad}(M)$ is $\operatorname{Rad}$-supplemented.

Proof. If $M$ is $\operatorname{Rad}$-supplemented, then $M$ is semilocal. Thus $\frac{M}{\operatorname{Rad}(M)}$ is reduced. By Proposition 2, $\operatorname{Rad}(M)$ is $\operatorname{Rad}$-supplemented . Conversely, suppose that $M$ is semilocal and $\operatorname{Rad}(M)$ is $\operatorname{Rad}$-supplemented. From Theorem 4 the assumption implies that $M$ is Rad-supplemented.

Using the above proposition we obtain the following characterization of semilocal rings.

Corollary 6. The following conditions on a ring $R$ is equivalent:

(1) $R$ is semilocal.

(2) Every left $R$-module with Rad-supplemented radical is Rad-supplemented.

Proof. (1) $\Rightarrow$ (2) If $R$ is semilocal, then every left $R$-module is semilocal by [7, Theorem 3.5]. The result follows from Proposition 3.

(2) $\Rightarrow$ (1) Since $\operatorname{Rad}\left(\frac{R}{\operatorname{Rad}(R)}\right)=0$, it follows from the hypothesis that $\frac{R}{\operatorname{Rad}(R)}$ is $\operatorname{Rad}$-supplemented. So $\frac{R}{\operatorname{Rad}(R)}$ is semisimple, i.e. $R$ is semilocal.

In [5], a module $M$ is said to be totally Rad-supplemented if every submodule of $M$ is Rad-supplemented. Every semisimple module is totally Rad-supplemented. It is easy to check that the class of totally Rad-supplemented modules is closed under factor modules and submodules. The following fact is a modification of Theorem 4 . 
Theorem 5. Let $M$ be a module and $\frac{M}{N}$ be reduced for some submodule $N$ of $M$. Then $M$ is totally Rad-supplemented if and only if $N$ and $\frac{M}{N}$ are totally Radsupplemented.

Proof. Suppose that $N$ and $\frac{M}{N}$ are totally Rad-supplemented. Let $U$ be any submodule of $M$. By the hypothesis, $U \cap N$ and $\frac{U+N}{N}$ are Rad-supplemented. Note that

$$
\frac{U+N}{N} \cong \frac{U}{U \cap N}
$$

is reduced because $\frac{M}{N}$ is reduced. By Theorem 4, $U$ is Rad-supplemented. Hence $M$ is totally Rad-supplemented.

Corollary 7. Let $M$ be a Rad-supplemented module. Then, $M$ is totally Radsupplemented if and only if $P(M)$ is totally $\mathrm{Rad}$-supplemented.

Proof. Suppose that $P(M)$ is totally Rad-supplemented. By the hypothes is and Corollary 3, $\frac{M}{P(M)}$ is supplemented. Applying [8, Proposition 2.6], we deduce that $\frac{M}{P(M)}$ is totally supplemented. Therefore $M$ is totally Rad-supplemented by Theorem 5.

\section{Rad-SUPPLEMENTED MODULES OVER COMMUTATIVE DOMAINS}

In this section a ring $R$ will be a commutative domain. Let $R$ be such a ring and $M$ be an $R$-module. We denote by $T(M)$ the set of all elements $m$ of $M$ for which there exists a non-zero element $r$ of $R$ such that $r m=0$, i.,e., Ann $(m) \neq 0$. Then $T(M)$, which is a submodule of $M$, called the torsion submodule of $M$. If $M=T(M)$, then $M$ is called a torsion module and $M$ is called torsion-free provided $T(M)=0$.

Proposition 4. Let $R$ be a non-semilocal commutative domain and $M$ be an $R$ module. If $M$ is totally $\mathrm{Rad}-$ supplemented, $M$ is a torsion module.

Proof. Let $0 \neq m \in M$. Suppose that $\operatorname{Ann}(m)=0$, i.e. $R \cong R m$. Since $M$ is totally Rad-supplemented, the left $R$-submodule $R m$ of $M$ is Rad-suppleme nted. So ${ }_{R} R$ is $\mathrm{Rad}$-supplemented. Therefore $\frac{R}{\operatorname{Rad}(R)}$ is semisimp le, i.e. $R$ is semilocal. This contradicts the assumption. Hence $\operatorname{Ann}(m) \neq 0$, this implies that $M$ is torsion.

Corollary 8. Let $R$ be a non-semilocal dedekind domain and $M$ be a totally Radsupplement ed $R$-module. Then $M$ is torsion.

Let $R$ be a dedekind domain and $M$ be an $R$-module. We denote by $\Omega$ the set of all maximal (i.e., prime) ideals of $R$. Suppose that $\mathfrak{p}$ is any element of $\Omega$. We denote by $T_{\mathfrak{p}}(M)$, which is a submodule of $M$, the set of all elements $m$ of $M$ for which there exists a positive integer $n$ such that $\mathfrak{p}^{n} m=0$. Then $T_{\mathfrak{p}}(M)$ is called the $\mathfrak{p}$-primary part of $M$. For a torsion module $M$ over a dedekind domain, we have the decomposition $M=\bigoplus_{\mathfrak{p} \in \Omega} T_{\mathfrak{p}}(M)$. 
Lemma 5. Let $R$ be a non-local dedekind domain and $M$ be an $R$-module. Then $M$ is $\operatorname{Rad}$-supplemented if and only if $\frac{M}{P(M)}$ is torsion and every $\mathfrak{p}$-primary part of $\frac{M}{P(M)}$ is (Rad-)supplemented.

Proof. According to [14, Theorem 3.1] and [5, Theorem 7.4], the proof of the lemma is clear.

Let $R$ be a dedekind domain and $M$ be an $R$-module. By [2, Lemma 4.4], $P(M)$ is injective and so there exists a direct summand $N$ of $M$ such that $\frac{M}{P(M)} \cong N$. This fact and Lemma 5 give the following basic result for torsion-free modules.

Corollary 9. Let $M$ be a torsion-free $\mathrm{Rad}$-supplemented module over a non-local dedekind domain. Then $M$ is radical.

Let $M$ be a radical module. $M$ is called simply radical if $M$ has no proper radical submodules.

Proposition 5. Let $R$ be a noetherian ring and $M$ be a simply radical $R$-module. If $M$ is amply $\mathrm{Rad}$-supplemented, $M$ is hollow radical. In particular, every $\mathrm{Rad}$ supplemented proper submodule of $M$ is supplemented.

Proof. Let $U$ be any proper submodule of $M$. Suppose that $U+V=M$ for some submodule $V$ of $M$. By the hypothesis, there exists a submodule $V^{\prime}$ of $V$ such that $U+V^{\prime}=M$ and $U \cap V^{\prime} \subseteq \operatorname{Rad}\left(V^{\prime}\right)$. Since $M$ is simply radical, it follows that $\operatorname{Rad}\left(V^{\prime}\right)=V^{\prime} \cap \operatorname{Rad}(M)=V^{\prime} \cap M=V^{\prime}$. So $V^{\prime}$ is radical. Therefore $V^{\prime}=M$ and so $V=M$. Then we deduce that $U$ is small in $M$. Hence $M$ is hollow radical. Suppose that a proper submodule $N$ of $M$ is Rad-supplemented. Since $M$ is simply radical, every submodule of $N$ contains a maximal submodule, i. e., $P(N)=0$. By Lemma $4, N$ is supplemented.

Corollary 10. Let $R$ be a dedekind domain and $M$ be a radical $R$-module. Then $M$ is amply Rad-supplemented and indecomposable if and only if the module is hollow radical.

Proof. Since indecomposable radical modules over dedekind domains is simply radical, $M$ is hollow radical by Proposition 5. The converse is clear.

Proposition 6. Let $M$ be a module over a Dedekind domain. Then the following statements are equivalent.

(1) $M$ is indecomposable, $w$-local and amply Rad-supplemented.

(2) $M$ is local.

Proof. (1) $\Rightarrow$ (2) Let $U$ be any proper submodule of $M$. Suppose that $U$ is not contained $\operatorname{Rad}(M)$. Since $M$ is w-local, $\operatorname{Rad}(M)$ is maximal and so $U+\operatorname{Rad}(M)=$ $M$. By the hypothesis, there exists a submodule $V$ of $\operatorname{Rad}(M)$ such that $U+V=M$ and $U \cap V \subseteq \operatorname{Rad}(V)$. It follows that $\operatorname{Rad}(V)=V \cap \operatorname{Rad}(M)=V$, i.e. $V$ is radical. 
Then, by [2, Lemma 4.4], $V$ is injective and so there exists a submodule $L$ of $M$ such that $M=V \oplus L$. Since $M$ is indecomposable and w-local, we get $V=0$. Thus, $U=M$, implying that $M$ is local.

(2) $\Rightarrow(1)$ is clear.

Now, we give an analogous characterization of [14, Theorem 3.1] for totally Radsupplemented modules.

Theorem 6. Let $M$ be a non-semilocal dedekind domain and $M$ be an R-module. Then $M$ is totally Rad-supplemented if and only if $M$ is torsion and every $\mathfrak{p}$-primary part of $M$ is totally Rad-supplemented.

Proof. The necessity of the condition is obvious by Corollary 8. Conversely, suppose that $M$ is torsion and every $\mathfrak{p}$-primary part of $M$ is totally Rad-supplemented. Let $N \subseteq U \subseteq M$. Since $M=\bigoplus_{\mathfrak{p} \in \Omega} T_{\mathfrak{p}}(M)$, we have $U=\bigoplus_{\mathfrak{p} \in \Omega}\left(U \cap T_{\mathfrak{p}}(M)\right)$ and $N=\bigoplus_{\mathfrak{p} \in \Omega}\left(N \cap T_{\mathfrak{p}}(M)\right)$. By the hypothesis, $N \cap T_{\mathfrak{p}}(M)$ has a Rad-supplement $V_{\mathfrak{p}}$ in $U \cap T_{\mathfrak{p}}(M)$. So $U \cap T_{\mathfrak{p}}(M)=N \cap T_{\mathfrak{p}}(M)+V_{\mathfrak{p}}$ and $N \cap V_{\mathfrak{p}} \subseteq \operatorname{Rad}\left(V_{\mathfrak{p}}\right)$. Let $V=\bigoplus_{\mathfrak{p} \in \Omega} V_{\mathfrak{p}}$. Then $N+V=U$. Since $N \cap V_{\mathfrak{p}} \subseteq \operatorname{Rad}\left(V_{\mathfrak{p}}\right)$ for every $\mathfrak{p} \in \Omega$, by [6, Corollaries 9.1.5 (c)], $N \cap V=\left(\bigoplus_{\mathfrak{p} \in \Omega}\left(N \cap T_{\mathfrak{p}}(M)\right)\right) \cap\left(\bigoplus_{\mathfrak{p} \in \Omega} V_{\mathfrak{p}}\right) \subseteq \operatorname{Rad}(V)$. Hence $U$ is Rad-supplemented. This completes the proof.

Finally, we give an example showing the class of (totally) Rad-supplemented modules is not closed under extensions, in general. For a module $M$, $\operatorname{Soc}(M)$ will indicate the sum of all simple submodules of $M$.

Example 2. (see [10, Example 2.3]) Consider the non-Noetherian commutative ring which is the direct product $\prod_{i \geq 1}^{\infty} F_{i}$, where $F_{i}=F$ is any field. Suppose that $R$ is the subring of the ring consisting of all sequences $\left(r_{n}\right)_{n \in \mathbb{N}}$ such that there exist $r \in F, m \in \mathbb{N}$ with $r_{n}=r$ for all $n \geq m$. Let $M={ }_{R} R$. Then $M$ is a regular module which is not semisimple. Therefore $\operatorname{Soc}(M)$ is a maximal submodule of $M$. This means that $\operatorname{Soc}(M)$ and $\frac{M}{\operatorname{Soc}(M)}$ are Rad-supplemented. On the other hand, $M$ is not Rad-supplemented.

\section{ACKNOWLEDGEMENT}

We would like to thank the referee for the valuable suggestions and comments which improved the revision of the paper.

\section{REFERENCES}

[1] K. Al-Takhman, C. Lomp, and R. Wisbauer, “ $\tau$-complemented and $\tau$-supplemented modules," Algebra Discrete Math., vol. 2006, no. 3, pp. 1-15, 2006.

[2] R. Alizade, G. Bilhan, and P. F. Smith, "Modules whose maximal submodules have supplements," Commun. Algebra, vol. 29, no. 6, pp. 2389-2405, 2001.

[3] R. Alizade and E. Büyükaşik, "Extensions of weakly supplemented modules," Math. Scand., vol. 103, no. 2, pp. 161-168, 2008. 
[4] E. Büyükaşik and C. Lomp, "On a recent generalization of semiperfect rings," Bull. Aust. Math. Soc., vol. 78, no. 2, pp. 317-325, 2008.

[5] E. Büyükasik, E. Mermut, and S. Özdemir, "Rad-supplemented modules," Rend. Semin. Mat. Univ. Padova, vol. 124, pp. 157-177, 2010.

[6] F. Kasch, Modules and rings, ser. London Mathematical Society Monographs, D. A. R. Wallace, Ed. London - New York: Academic Press, 1982, vol. 17.

[7] C. Lomp, "On semilocal modules and rings," Commun. Algebra, vol. 27, no. 4, pp. 1921-1935, 1999.

[8] P. Rudlof, "On the structure of couniform and complemented modules," J. Pure Appl. Algebra, vol. 74, no. 3, pp. 281-305, 1991.

[9] D. W. Sharpe and P. Vamos, Injective modules, ser. Cambridge Tracts in Mathematics and mathematical Physics. Cambridge: University Press, 1972, vol. 62.

[10] P. F. Smith, "Finitely generated supplemented modules are amply supplemented," The Arabian Journal for Science And Engineering, vol. 25, no. 2C, pp. 69-79, 2000.

[11] E. Türkmen and A. Pancar, "On cofinitely Rad-supplemented modules," Int. J. Pure Appl. Math., vol. 53, no. 2, pp. 153-162, 2009.

[12] R. Wisbauer, Foundations of module and ring theory. A handbook for study and research, ser. Algebra, Logic and Applications. Philadelphia: Gordon and Breach Science Publishers, 1991, vol. 3.

[13] W. Xue, "Characterizations of semiperfect and perfect rings," Publ. Mat., Barc., vol. 40, no. 1, pp. 115-125, 1996.

[14] H. Zöschinger, “Komplementierte moduln über Dedekindringen,” J. Algebra, vol. 29, pp. 42-56, 1974.

[15] H. Zöschinger, “Koatomare moduln,” Math. Z., vol. 170, pp. 221-232, 1980.

Authors' addresses

Ergül Türkmen

Ondokuz Mayıs University, Faculty of Art and Science, Department of Mathematics, 55139, Samsun, Turkey

E-mail address: eturkmen@omu.edu.tr

\section{Ali Pancar}

Ondokuz Mayis University, Faculty of Art and Science, Department of Mathematics, 55139, Samsun, Turkey

E-mail address: apancar@omu.edu.tr 